\title{
Efficacy of Plant Extracts, Bioagents and Fungicides against Fusarium udum Causing Pigeonpea Wilt
}

\author{
Sandeep Kumar ${ }^{1 *}$, Divya Singh ${ }^{1}$, Jay Kumar Yadav ${ }^{1}$, \\ Susheel Kumar ${ }^{1}$ and Sandeep Kumar Verma ${ }^{2}$ \\ ${ }^{1}$ Department of Plant Pathology, Narendra Deva University of Agriculture and Technology \\ Kumarganj -Faizabad, Uttar Pradesh (224229), India \\ ${ }^{2}$ IASRI, New Delhi, India \\ *Corresponding author
}

A B S T R A C T

Keywords

Fusarium,

Pigeonpea, Wilt,

Plant extracts,

Bioagent.

Article Info

Accepted:

26 August 2017

Available Online:

10 September 2017
Pigeonpea [Cajanus cajan (L.) Millsp.] is the most important pulse crop of the India. It is suffered number of diseases like, Alternaria leaf spot, Phytophthora blight, Sterility mosaic and wilt. Pigeonpea wilt is a most destructive soil borne disease caused by $F$. udum. It is wide spread and caused heavy yeild loss nearly $100 \%$. Plant extracts, bioagents and fungicides were evaluated against Fusarium udum. Out of seventeen treatments tested in vitro, propiconazole $(0.15 \%)$ was found most effective in inhibiting radial growth of the pathogen followed by copper oxychloride, Garlic, Garlic + Tulsi, Tulsi, T. viride, Ginger + Mehandi, Neem, Onion, A. niger, Ginger, Onion + Beal, Mehandi, Beal, Neem + Marigold, $P$. notatum and Marigold over the check. The highest percent disease control was 97.22 to 94.44 in Garlic and lowest 58.33 to 49.44 in Marigold. Among the bio-agents seed treatment with $T$. viride had controlled maximum wilt incidence 47.22 to $88.88 \%$ followed by $A$. niger (41.66 to $77.78 \%$ ) and P. notatum (25.00 to $61.11 \%$ ). Both fungicides were highly effective in reducing the disease incidence. The effectiveness was $100 \%$ in propiconazole and 97.22 to $100 \%$ in copper oxychloride.

\section{Introduction}

Pulses play a major role in nutritional security for the people, particularly those depends on cereal based diet and also play an important role in sustaining intensive agriculture by improving physical, chemical and biological properties of soil and are considered excellent crop for diversification of cereal based cropping system. Pigeonpea [Cajanus cajan (L.) Millsp.] is the multipurpose legume crop with diversified uses as food, feed, fodder and fuel. It has been recognized as a valuable source of protein (17.9 to 24.39/100g) particularly in the developing countries where majority of the population depends on the vegetarian foods for meeting its dietary requirements (Ali and Kumar, 2005). It was grown on about 3.53 million ha area producing 2.43 million tonnes with production of $697 \mathrm{~kg} / \mathrm{ha}$. In U.P. it is grown in 0.33 million ha area producing 0.30 million tones with productivity of $9.14 \mathrm{q} / \mathrm{ha}$ (Anonymous, 2011).

India is the largest producer and consumer of pulses in the world and also key player with $25 \%$ share in the global basket from an area 
about 33\% (Ali, 2007). The yield of pigeonpea is greatly affected by wilt disease caused by fungi Fusarium udum. Pigeonpea wilt is an important disease in India, Kenya, Malawi, Nepal, Tanzania and Uganda (Reddy et al., 1990). In India, it is the most serious problem all over the pigeonpea growing areas especially in U.P, M.P, Bihar and Maharashtra. However, loss in individual plant found nearly $100 \%$ when wilt occurred at pre-podding stage, $67 \%$ at podding stage and $29.5 \%$ at pre-harvest stage (Kannaiyan et al., 1981). Fusarium udum is a host specific pathogen of pigeonpea. The fungus is primarily a soil borne facultative parasite and enters the host through fine roots and subsequently colonizes in different plant parts (Khune, 1990). In discriminate use of these chemicals has led to development of fungicide resistance strain (Okigbo, 2004) and more importantly, environmental pollution, posing a potential risk to animal and human health (Lyon et al., 1995). Plant extracts and bioagents are emerging as eco- friendly way to manage pathogen. So, the objective of this investigation was to evaluate the potential of plant extracts, bioagents and fungicides against Fusarium udum.

\section{Materials and Methods}

\section{Isolation}

Pigeonpea plants showing characteristic symptoms of Fusarium wilt were collected from pigeonpea experimental field, Department of Genetics and Plant Breeding, NDUA\&T, Kumarganj, Faizabad for isolation and identification. The infected plant parts were cut in to small pieces and surface sterilized with 0.1 per cent Mercuric Chloride solution and washed thoroughly 3 to 4 times with sterilized water to remove the traces of Mercuric Chloride. The pieces were transferred in Petri dishes containing potato Dextrose Agar and incubated at $25^{\circ} \mathrm{C}$ for 6 days. Pure colonies are isolated from inoculated petriplates separately in aseptic condition.

\section{Efficacy of plant extract against Fusarium wilt in vitro}

In order to find out the efficacy of various plant extract against the Fusarium wilt 8 plant extracts viz., leaves of Neem, Mehandi, Tulsi, Beal, Marigold, bulb of Garlic, Onion and Ginger were used. Fresh leaves, bulb and rhizome were collected and washed thoroughly in clean water. $100 \mathrm{~g}$ of each washed plant material was grinded in Pestle and Mortar by adding equal amount $(100 \mathrm{ml})$ of sterilized water $(1: 1 \mathrm{~V} / \mathrm{W})$ and heated at 80 ${ }^{0} \mathrm{C}$ for 10 minutes in hot water both. The materials was filtered through double layered muslin cloth followed by filtering through sterilized What man No. 1 filter paper and treated as standard plant extract $(100 \%)$. The stock solution $5 \%$ and $10 \%$ concentration were made by adding 90 and $95 \mathrm{ml}$ of sterilized PDA media to obtained 5 and $10 \%$ concentration of plant extract.

To study the inhibitory effect of botanicals on mycelial growth of $F$. udum, 5 and $10 \%$ concentration were used by applying poison food techniques under in vitro condition. Seventeen treatments having four replications were maintained. Five mm discs of 7 days old culture of $F$. udum were cut with sterilized cork borer and placed in the centre of plant extract amended petriplates. The control petriplates having PDA alone were inoculated in the same manner. These petriplates were incubated at $25 \pm 2{ }^{0} \mathrm{C}$. The observations were recorded on radial growth at $48 \mathrm{hrs}, 72,96$, 120 and $144 \mathrm{hrs}$ of incubation.

\section{Efficacy of plant extracts against Fusarium udum in vivo}

The concentration of plant extracts found effective in vitro will be further tested in vivo. Soil was autoclaved, filled in $20 \mathrm{~cm}$ diameter 
earthen pots separately @ 4 kg per pot and inoculation was done by adding $F$. udum grown on wheat grain medium @ 5\% of the weight of soil in the pots. Control pots were filled with soil without adding inoculum. Seven days after inoculation of pathogen, $10 \%$ concentration of plant extracts @ $100 \mathrm{ml}$ per $\mathrm{kg}$ of soil was thoroughly mixed in the pots to determine the effect of plant extract in vivo.

The seeds of pigeonpea wilt susceptible variety Bahar were sown in each inoculated pot (15 seed per pot), where finally 12 plants were maintained. The experiment was conducted in CRD with 18 treatments including control.

First appearance of disease, disease incidence and per cent disease control were observed 45, 60, 75, 90, 105 and 120 days after sowing.

\section{Efficacy of bioagents against Fusarium udum in vitro}

The rhizospheric fungi of pigeonpea namely Trichoderma viride, Aspergillus niger and Penicillium notatum were isolated from pigeonpea grown in the G.P.B. research farm of N.D.U.A. \& T, Kumarganj, Faizabad, U.P. These dominant rhizospheric fungi were isolated by soil plate methods as described by Dhingra and Sinclair (1995) using Martin's agar medium. The rhizospheric fungi were screened for their antagonistic potential against $F$. udum by the method described by Upadhyay and Rai (1987). 5mm agar block of 5 day old culture of pathogen and bio-agents were used for test as dual culture methods. The experiments was performed in four replications for each treatments, for the control set, the same size of agar block of pathogen was put cooled distilled $16 \mathrm{ml}$ of PDA medium. The radial colony growth was measured after 48, 72, 96, 120 and $144 \mathrm{hrs}$ of incubation.

\section{Efficacy of bioagents against Fusarium} udum in vivo

The mass culture of the antagonists and the pathogen was prepared on wheat grains following the methods of Singh et al., (1996). The sterilized soil was well mixed and separately with $1 \%(\mathrm{v} / \mathrm{w})$ pure inoculum of the pathogen.

The pathogen infested soil was placed in plastic pots $(25 \times 20 \mathrm{~cm})$ and kept at room temperature for 7 days to allow the pathogen to establish well in the soil. The pure inoculum $5 \%$ of $T$. viride, $A$. niger and $P$. notatum were mixed separately with the pathogen infested soil. The pots containing the soil pathogen inocula without the antagonist served as control. Four replications were maintained for each treatment. The $50 \%$ soil moisture was maintained by adding water once a day. Disease development was observed regularly. The final record of the incidence of wilting in plants was done at 45 , 60, 75, 90, 105 and 120 days after sowing.

\section{Efficacy of fungicides against Fusarium wilt in vitro}

The fungicides viz., Propiconazole (0.15\%) and Copper oxychloride $(0.30 \%)$ were brought from authentic dealer. The required amount of Propiconazole $0.15 \mathrm{ml} / 100 \mathrm{ml}$ of PDA and Copper oxychloride $0.30 \mathrm{~g} / 100 \mathrm{ml}$ PDA were mixed in the flask shacked and poured in petriplates replicated. The standard technique was followed for testing of mycelium growth of pathogen.

\section{Efficacy of fungicides against Fusarium wilt in vivo}

Fungicides that showed good inhibitory effect in vitro were selected for seed treatment in vivo. Seeds of pigeonpea variety "Bahar" were moist for $12 \mathrm{hrs}$ prior to showing and 
then treated with the Propiconazole $(0.15 \%)$ and Copper oxychloride $(0.30 \%)$. Twenty (20) treated seeds of susceptible variety "Bahar" were shown in each wilt sick pot and only 12 plants are maintained after germination. Percent wilt incidence was recorded at 45, 60, 75, 90, 105 and120 days after showing.

The percent growth inhibition, percent disease incidence and percent disease control were calculated by using the following formula

$$
\mathrm{I}=\frac{\mathrm{C}-\mathrm{T}}{\mathrm{C}} \times 100
$$

Where,

$I=$ Per cent inhibition of fungal growth

$\mathrm{C}=$ Radial growth of control

$\mathrm{T}=$ Radial growth of treated petriplates

Per cent disease incidence $=\frac{\text { Number of infected plants }}{\text { Total number of plants }} \times 100$

Per cent disease control $=\frac{\mathrm{C}-\mathrm{T}}{\mathrm{C}} \times 100$

Where,

$\mathrm{C}=$ Per cent disease incidence of control plots

$\mathrm{T}=$ Per cent disease incidence in treated plots

\section{Results and Discussion}

Efficacy of plant extracts, chemicals and bio-agents in vitro

During the present investigation of seventeen treatments namely, Neem, Garlic, Onion, Ginger, Marigold, Tulsi, Beal, Mehandi, Neem + Marigold, Garlic + Tulsi, Onion + Beal, Ginger + Mehandi, T. viride, A. niger, $P$. notatum, Propiconazole and Copper oxychloride were evaluated for fungi toxicity against Fusarium udum by using poison food technique. The results (Tables 1 and 2) showed that all treatments inhibited the mycelia growth of $F$. udum. The effectiveness of the extracts increased with an increasing concentration. The maximum inhibition was recorded at $10 \%$ concentration.

Devi and Charley (2012) reported the effect of different plant extracts against the mycelial growth of $F$. udum. Among them extract of $A$. sativum showed complete inhibition of radial growth of $F$. udum followed by $A$. indica (74.4\%), Spilanthes acemella $(68.8 \%)$ and Aloe very $(55.9 \%)$.

Two fungicides namely propiconazole $(0.15 \%)$ and copper oxychloride $(0.30 \%)$ were tested by poisoned food technique in vitro and resulted cent percent restriction of radial growth of $F$. udum up to $144 \mathrm{hrs}$. Mehta et al., (2010) reported laboratory test that the propiconazole (1000 \& $1500 \mathrm{ppm})$ and copper oxychloride (3000 ppm) had inhibited cent percent growth of the $F$. udum upto 7 days.

It is found from the experiment that Garlic extract inhibit the growth of $F$. udum up to 76.87 to $83.22 \%$ at $5 \%$ concentration and 85.41 to $90.37 \%$ at $10 \%$ concentration followed by Garlic + Tulsi (80.20 to 88.14\%) up to 144 hrs incubation. Mehta et al., (2010) found the Garlic bulb extract was significantly superior to inhibit the growth of F. udum.

The growth of $F$. udum reduced from $72.96 \%$ to $81.27 \%$ at $5 \%$ concentration and $73.95 \%$ to $85.92 \%$ at $10 \%$ concentration up to $144 \mathrm{hrs}$ incubation followed by Neem (54.21-74.41\%, 61.00-79.25\%), Onion (49.21-70.77\%, 55.20$74.81 \%)$, Ginger $\quad(41.87-68.55 \%, 52.00-$ $72.96 \%)$, Mehandi (38.90-66.11\%, 30.55$66.66 \%)$, Beal (31.09-64.11\%, 33.33-69.44\%) and Marigold (26.87-62.27\%, 41.66-77.78\%). The mycelial growth inhibition rate was increased with increasing concentration of 
plant extracts. Baby Joseph et al., (2008) tested different concentration i.e, 5\%, 10\%, $15 \%$ and $20 \%$ extracts of different plant viz., Artemisia annua, Eucalyptus globules, $O$. sanctum and Rheum emodi and found significant reduction in growth of $F$. solani. Plant extracts were found most effective at
$20 \%$ concentration. The mixture extract of Ginger + Mehandi inhibited the germination of $F$. udum from 66.37 to $80.37 \%$ followed by Onion + Beal (48.95-70.37\%) and neem + marigold (36.45-65.18\%) from $48 \mathrm{hrs}$ to 144 hrs. incubation period.

Table.1 Effect of plant extracts (5\% conc. each) on the per cent mycelial Growth inhibition of $F$. udum

\begin{tabular}{|c|c|c|c|c|c|}
\hline \multirow[t]{2}{*}{ Treatments } & \multicolumn{5}{|c|}{ Per cent inhibition } \\
\hline & $48 \mathrm{hrs}$ & $72 \mathrm{hrs}$ & $96 \mathrm{hrs}$ & 120 hrs & 144 hrs \\
\hline Neem & $\begin{array}{l}54.21 \\
(47.14)\end{array}$ & $\begin{array}{l}59.88 \\
(50.73)\end{array}$ & $\begin{array}{l}66.33 \\
(54.57)\end{array}$ & $\begin{array}{l}72.56 \\
(58.44)\end{array}$ & $\begin{array}{l}74.61 \\
(59.77)\end{array}$ \\
\hline Garlic & $\begin{array}{l}76.87 \\
(61.28)\end{array}$ & $\begin{array}{l}78.33 \\
(62.28)\end{array}$ & $\begin{array}{l}80.17 \\
(63.58)\end{array}$ & $\begin{array}{l}82.76 \\
(65.48)\end{array}$ & $\begin{array}{l}83.22 \\
(65.82)\end{array}$ \\
\hline Onion & $\begin{array}{l}49.21 \\
(44.55)\end{array}$ & $\begin{array}{l}56.07 \\
(48.48)\end{array}$ & $\begin{array}{l}63.48 \\
(52.82)\end{array}$ & $\begin{array}{l}68.02 \\
(55.56)\end{array}$ & $\begin{array}{l}70.77 \\
(57.27)\end{array}$ \\
\hline Ginger & $\begin{array}{l}41.87 \\
(40.31)\end{array}$ & $\begin{array}{l}51.66 \\
(45.95)\end{array}$ & $\begin{array}{l}60.17 \\
(50.87)\end{array}$ & $\begin{array}{l}65.39 \\
(53.97)\end{array}$ & $\begin{array}{l}68.55 \\
(55.40)\end{array}$ \\
\hline Marigold & $\begin{array}{l}26.87 \\
(31.16)\end{array}$ & $\begin{array}{l}40.59 \\
(39.54)\end{array}$ & $\begin{array}{l}48.30 \\
(44.02)\end{array}$ & $\begin{array}{l}59.27 \\
(50.34)\end{array}$ & \begin{tabular}{|l}
62.22 \\
$(52.52)$
\end{tabular} \\
\hline Tulsi & $\begin{array}{l}72.96 \\
(58.69)\end{array}$ & $\begin{array}{l}74.16 \\
(59.46)\end{array}$ & $\begin{array}{l}77.05 \\
(61.38)\end{array}$ & $\begin{array}{l}80.46 \\
(63.77)\end{array}$ & $\begin{array}{l}81.27 \\
(64.36)\end{array}$ \\
\hline Beal & $\begin{array}{l}31.09 \\
(33.86)\end{array}$ & $\begin{array}{l}42.26 \\
(40.54)\end{array}$ & $\begin{array}{l}53.12 \\
(46.79)\end{array}$ & $\begin{array}{l}60.13 \\
(50.84)\end{array}$ & $\begin{array}{l}64.11 \\
(53.20)\end{array}$ \\
\hline Mehandi & $\begin{array}{l}38.90 \\
(38.57)\end{array}$ & $\begin{array}{l}47.97 \\
(4383)\end{array}$ & $\begin{array}{l}53.57 \\
(47.05)\end{array}$ & $\begin{array}{l}62.50 \\
(52.24)\end{array}$ & $\begin{array}{l}66.11 \\
(54.40)\end{array}$ \\
\hline Control & $\begin{array}{l}0.00 \\
(0.00)\end{array}$ & $\begin{array}{l}0.00 \\
(0.00)\end{array}$ & $\begin{array}{l}0.00 \\
(0.00)\end{array}$ & $\begin{array}{l}0.00 \\
(0.00)\end{array}$ & $\begin{array}{l}0.00 \\
(0.00)\end{array}$ \\
\hline $\mathrm{CD}$ at $5 \%$ & 6.01 & 5.10 & 3.30 & 2.55 & 2.15 \\
\hline
\end{tabular}


Table. 2 Effect of plant extracts (10\% conc. each), chemicals and bio-agents on the per cent Mycelial growth inhibition of $F$. udum

\begin{tabular}{|c|c|c|c|c|c|}
\hline \multirow[t]{2}{*}{ Treatments } & \multicolumn{5}{|c|}{ Per cent inhibition } \\
\hline & 48 hrs & $72 \mathrm{hrs}$ & $96 \mathrm{hrs}$ & 120 hrs & 144 hrs \\
\hline Neem & $\begin{array}{l}61.45 \\
(51.60)\end{array}$ & $\begin{array}{l}65.07 \\
(53.75)\end{array}$ & $\begin{array}{l}72.01 \\
(58.04)\end{array}$ & $\begin{array}{l}78.06 \\
(62.05)\end{array}$ & $\begin{array}{l}79.25 \\
(62.87)\end{array}$ \\
\hline Garlic & $\begin{array}{l}85.41 \\
(67.53)\end{array}$ & $\begin{array}{l}86.25 \\
(68.22)\end{array}$ & $\begin{array}{l}88.09 \\
(69.67)\end{array}$ & $\begin{array}{l}89.90 \\
(71.45)\end{array}$ & $\begin{array}{l}90.37 \\
(71.89)\end{array}$ \\
\hline Onion & $\begin{array}{l}55.20 \\
(47.96)\end{array}$ & $\begin{array}{l}60.31 \\
(50.93)\end{array}$ & $\begin{array}{l}67.85 \\
(55.44)\end{array}$ & $\begin{array}{l}71.92 \\
(57.98)\end{array}$ & $\begin{array}{l}74.81 \\
(59.85)\end{array}$ \\
\hline Ginger & $\begin{array}{l}52.08 \\
(46.17)\end{array}$ & $\begin{array}{l}58.72 \\
(50.00)\end{array}$ & $\begin{array}{l}64.87 \\
(53.63)\end{array}$ & $\begin{array}{l}67.97 \\
(55.51)\end{array}$ & $\begin{array}{l}72.96 \\
(58.64)\end{array}$ \\
\hline Marigold & $\begin{array}{l}30.20 \\
(33.32)\end{array}$ & $\begin{array}{l}36.25 \\
(37.00)\end{array}$ & $\begin{array}{l}47.61 \\
(43.61)\end{array}$ & $\begin{array}{l}58.76 \\
(50.02)\end{array}$ & $\begin{array}{l}61.96 \\
(52.49)\end{array}$ \\
\hline Tulsi & $\begin{array}{l}73.95 \\
(59.29) \\
\end{array}$ & $\begin{array}{l}77.77 \\
(61.85) \\
\end{array}$ & $\begin{array}{l}80.94 \\
(64.09)\end{array}$ & $\begin{array}{l}84.64 \\
(66.90) \\
\end{array}$ & $\begin{array}{l}85.92 \\
(67.93) \\
\end{array}$ \\
\hline Beal & $\begin{array}{l}40.62 \\
(39.57) \\
\end{array}$ & $\begin{array}{l}46.81 \\
(43.15)\end{array}$ & $\begin{array}{l}55.35 \\
(48.05)\end{array}$ & $\begin{array}{l}62.71 \\
(52.34) \\
\end{array}$ & $\begin{array}{l}66.29 \\
(54.48) \\
\end{array}$ \\
\hline Mehandi & $\begin{array}{l}47.91 \\
(43.78) \\
\end{array}$ & $\begin{array}{l}51.58 \\
(45.89) \\
\end{array}$ & $\begin{array}{l}57.14 \\
(49.08) \\
\end{array}$ & $\begin{array}{l}64.03 \\
(53.12) \\
\end{array}$ & $\begin{array}{l}67.40 \\
(55.16) \\
\end{array}$ \\
\hline Neem + Marigold & $\begin{array}{l}36.45 \\
(37.12) \\
\end{array}$ & $\begin{array}{l}44.43 \\
(41.78) \\
\end{array}$ & $\begin{array}{l}52.37 \\
(46.34) \\
\end{array}$ & $\begin{array}{l}61.40 \\
(51.56) \\
\end{array}$ & $\begin{array}{l}65.18 \\
(53.81) \\
\end{array}$ \\
\hline Garlic + Tulsi & $\begin{array}{l}80.20 \\
(63.56) \\
\end{array}$ & $\begin{array}{l}82.53 \\
(65.27) \\
\end{array}$ & $\begin{array}{l}85.11 \\
(67.28) \\
\end{array}$ & $\begin{array}{l}87.27 \\
(69.07) \\
\end{array}$ & $\begin{array}{l}88.14 \\
(69.83) \\
\end{array}$ \\
\hline Onion + Beal & $\begin{array}{l}48.95 \\
(44.38)\end{array}$ & $\begin{array}{l}55.15 \\
(47.93) \\
\end{array}$ & $\begin{array}{l}60.71 \\
(51.16)\end{array}$ & $\begin{array}{l}65.80 \\
(58.54)\end{array}$ & $\begin{array}{l}70.37 \\
(56.99)\end{array}$ \\
\hline Ginger + Mehandi & $\begin{array}{l}66.33 \\
(54.51) \\
\end{array}$ & $\begin{array}{l}69.83 \\
(56.66) \\
\end{array}$ & $\begin{array}{l}73.80 \\
(59.19) \\
\end{array}$ & $\begin{array}{l}78.94 \\
(62.65) \\
\end{array}$ & $\begin{array}{l}80.37 \\
(63.67) \\
\end{array}$ \\
\hline T. viride & $\begin{array}{l}67.70 \\
(55.35) \\
\end{array}$ & $\begin{array}{l}71.42 \\
(57.66) \\
\end{array}$ & $\begin{array}{l}76.18 \\
(60.76) \\
\end{array}$ & $\begin{array}{l}79.38 \\
(62.97) \\
\end{array}$ & $\begin{array}{l}81.48 \\
(64.48) \\
\end{array}$ \\
\hline A. niger & $\begin{array}{l}53.12 \\
(46.77) \\
\end{array}$ & $\begin{array}{l}59.52 \\
(50.47) \\
\end{array}$ & $\begin{array}{l}66.66 \\
(54.71) \\
\end{array}$ & $\begin{array}{l}70.61 \\
(57.14) \\
\end{array}$ & $\begin{array}{l}72.59 \\
(58.40)\end{array}$ \\
\hline P. notatum & $\begin{array}{l}32.29 \\
(34.61) \\
\end{array}$ & $\begin{array}{l}39.67 \\
(39.02) \\
\end{array}$ & $\begin{array}{l}50.59 \\
(45.32) \\
\end{array}$ & $\begin{array}{l}60.08 \\
(50.79) \\
\end{array}$ & $\begin{array}{l}63.70 \\
(52.93) \\
\end{array}$ \\
\hline Propiconazole $(0.15 \%)$ & $\begin{array}{l}100.00 \\
(90.00) \\
\end{array}$ & $\begin{array}{r}100.00 \\
(90.00) \\
\end{array}$ & $\begin{array}{l}100.00 \\
(90.00) \\
\end{array}$ & $\begin{array}{l}100.00 \\
(90.00) \\
\end{array}$ & $\begin{array}{l}100.00 \\
(90.00) \\
\end{array}$ \\
\hline Copper oxychloride $(0.30 \%)$ & $\begin{array}{l}100.00 \\
(90.00) \\
\end{array}$ & $\begin{array}{l}100.00 \\
(90.00) \\
\end{array}$ & $\begin{array}{l}100.00 \\
(90.00) \\
\end{array}$ & $\begin{array}{l}100.00 \\
(90.00) \\
\end{array}$ & $\begin{array}{l}100.00 \\
(90.00) \\
\end{array}$ \\
\hline Check & $\begin{array}{l}0.00 \\
(0.00) \\
\end{array}$ & $\begin{array}{l}0.00 \\
(0.00) \\
\end{array}$ & $\begin{array}{l}0.00 \\
(0.00) \\
\end{array}$ & $\begin{array}{l}0.00 \\
(0.00) \\
\end{array}$ & $\begin{array}{l}0.00 \\
(0.00) \\
\end{array}$ \\
\hline $\mathrm{CD}$ at $5 \%$ & 1.79 & 1.12 & 1.26 & 1.05 & 0.85 \\
\hline
\end{tabular}


Table.3 Effect of plant extracts (10\% conc. each), chemicals and bio-agents on the per cent Disease control Fusarium wilt of pigeon pea

\begin{tabular}{|c|c|c|c|c|c|c|}
\hline \multirow[t]{2}{*}{ Treatments } & \multicolumn{6}{|c|}{ Percent disease Control } \\
\hline & 45 day & 60 day & 75 day & 90 day & 105 day & 120 day \\
\hline Neem & $\begin{array}{l}83.33 \\
(65.87)\end{array}$ & $\begin{array}{l}75.00 \\
(59.97)\end{array}$ & $\begin{array}{l}66.66 \\
(54.71)\end{array}$ & $\begin{array}{l}58.33 \\
(48.77)\end{array}$ & $\begin{array}{l}50.00 \\
(44.98)\end{array}$ & $\begin{array}{l}44.44 \\
(42.98)\end{array}$ \\
\hline Garlic & $\begin{array}{l}97.22 \\
(83.38)\end{array}$ & $\begin{array}{l}94.44 \\
(78.17)\end{array}$ & $\begin{array}{l}91.66 \\
(73.18) \\
\end{array}$ & $\begin{array}{l}83.33 \\
(65.87)\end{array}$ & $\begin{array}{l}75.00 \\
(59.97)\end{array}$ & $\begin{array}{l}66.66 \\
(53.47)\end{array}$ \\
\hline Onion & $\begin{array}{l}80.55 \\
(63.88)\end{array}$ & $\begin{array}{l}72.22 \\
(58.20)\end{array}$ & \begin{tabular}{|l|}
63.88 \\
$(53.05)$
\end{tabular} & $\begin{array}{l}55.55 \\
(48.17)\end{array}$ & $\begin{array}{l}47.22 \\
(43.38)\end{array}$ & $\begin{array}{l}41.66 \\
(38.94)\end{array}$ \\
\hline Ginger & $\begin{array}{l}75.00 \\
(59.97)\end{array}$ & $\begin{array}{l}63.89 \\
(53.17)\end{array}$ & \begin{tabular}{|l|}
58.33 \\
$(49.77)$
\end{tabular} & $\begin{array}{l}50.00 \\
(44.98)\end{array}$ & $\begin{array}{l}44.44 \\
(42.98)\end{array}$ & $\begin{array}{l}38.88 \\
(39.74)\end{array}$ \\
\hline Marigold & $\begin{array}{l}58.33 \\
(49.77)\end{array}$ & $\begin{array}{l}49.44 \\
(56.45)\end{array}$ & \begin{tabular}{|l|}
41.66 \\
$(40.18)$
\end{tabular} & $\begin{array}{l}33.33 \\
(35.24)\end{array}$ & $\begin{array}{l}33.33 \\
(35.24)\end{array}$ & $\begin{array}{l}22.22 \\
(25.11)\end{array}$ \\
\hline Tulsi & $\begin{array}{l}91.66 \\
(73.18)\end{array}$ & $\begin{array}{l}83.33 \\
(65.87)\end{array}$ & \begin{tabular}{|l|}
75.00 \\
$(59.97)$ \\
\end{tabular} & $\begin{array}{l}66.66 \\
(54.71)\end{array}$ & $\begin{array}{l}58.33 \\
(48.57) \\
\end{array}$ & $\begin{array}{l}50.00 \\
(44.98)\end{array}$ \\
\hline Beal & $\begin{array}{l}66.66 \\
(54.79)\end{array}$ & $\begin{array}{l}55.55 \\
(48.17)\end{array}$ & \begin{tabular}{|l|}
50.00 \\
$(44.98)$
\end{tabular} & $\begin{array}{l}41.66 \\
(40.18)\end{array}$ & $\begin{array}{l}38.89 \\
(38.51)\end{array}$ & $\begin{array}{l}30.55 \\
(33.50)\end{array}$ \\
\hline Mehandi & $\begin{array}{l}69.44 \\
(56.53)\end{array}$ & $\begin{array}{l}58.33 \\
(49.81)\end{array}$ & \begin{tabular}{|l|}
52.77 \\
$(46.57)$
\end{tabular} & $\begin{array}{l}44.44 \\
(42.98)\end{array}$ & $\begin{array}{l}41.66 \\
(40.18)\end{array}$ & $\begin{array}{l}33.33 \\
(35.24)\end{array}$ \\
\hline Neem + Marigold & $\begin{array}{l}63.88 \\
(53.05)\end{array}$ & $\begin{array}{l}52.78 \\
(46.57)\end{array}$ & \begin{tabular}{|l|}
47.22 \\
$(43.38)$
\end{tabular} & $\begin{array}{l}38.89 \\
(38.51)\end{array}$ & $\begin{array}{l}38.89 \\
(38.51)\end{array}$ & $\begin{array}{l}27.78 \\
(31.75)\end{array}$ \\
\hline Garlic + Tulsi & $\begin{array}{l}94.44 \\
(78.17)\end{array}$ & $\begin{array}{l}91.66 \\
(73.18)\end{array}$ & \begin{tabular}{|l|}
83.33 \\
$(65.87)$
\end{tabular} & $\begin{array}{l}75.00 \\
(59.97)\end{array}$ & $\begin{array}{l}66.66 \\
(54.71)\end{array}$ & $\begin{array}{l}58.33 \\
(48.57)\end{array}$ \\
\hline Onion + Beal & $\begin{array}{l}72.22 \\
(58.44)\end{array}$ & $\begin{array}{l}61.11 \\
(51.45)\end{array}$ & \begin{tabular}{|l|}
55.55 \\
$(48.20)$ \\
\end{tabular} & $\begin{array}{l}47.22 \\
(43.38) \\
\end{array}$ & $\begin{array}{l}44.44 \\
(41.75)\end{array}$ & $\begin{array}{l}33.33 \\
(35.24)\end{array}$ \\
\hline Ginger + Mehandi & $\begin{array}{l}86.11 \\
(68.25)\end{array}$ & $\begin{array}{l}77.78 \\
(61.92)\end{array}$ & \begin{tabular}{|l|}
69.44 \\
$(56.45)$
\end{tabular} & $\begin{array}{l}61.11 \\
(51.45)\end{array}$ & $\begin{array}{l}52.78 \\
(46.57)\end{array}$ & $\begin{array}{l}44.44 \\
(42.98)\end{array}$ \\
\hline T. viride $(4 \mathrm{~g} / \mathrm{kg}$ seed $)$ & $\begin{array}{l}88.88 \\
(70.68)\end{array}$ & $\begin{array}{l}80.55 \\
(63.88)\end{array}$ & \begin{tabular}{|l|}
72.22 \\
$(58.20)$ \\
\end{tabular} & $\begin{array}{l}63.88 \\
(53.05) \\
\end{array}$ & $\begin{array}{l}55.55 \\
(48.17) \\
\end{array}$ & $\begin{array}{l}47.22 \\
(40.95)\end{array}$ \\
\hline A. niger $(4 \mathrm{~g} / \mathrm{kg}$ seed $)$ & $\begin{array}{l}77.78 \\
(61.92)\end{array}$ & $\begin{array}{l}66.66 \\
(54.71)\end{array}$ & \begin{tabular}{|l|}
61.11 \\
$(51.41)$
\end{tabular} & $\begin{array}{l}52.78 \\
(46.57)\end{array}$ & $\begin{array}{l}46.66 \\
(40.18)\end{array}$ & $\begin{array}{l}41.66 \\
(38.94)\end{array}$ \\
\hline P. notatum $(4 \mathrm{~g} / \mathrm{kg}$ seed $)$ & $\begin{array}{l}61.11 \\
(51.41)\end{array}$ & $\begin{array}{l}50.00 \\
(44.98)\end{array}$ & \begin{tabular}{|l|}
44.44 \\
$(41.78)$
\end{tabular} & $\begin{array}{l}36.11 \\
(36.78)\end{array}$ & $\begin{array}{l}36.11 \\
(36.78)\end{array}$ & $\begin{array}{l}25.00 \\
(49.98)\end{array}$ \\
\hline Propiconazole $(0.15 \%)$ & $\begin{array}{l}100.00 \\
(90.00)\end{array}$ & $\begin{array}{l}100.00 \\
(90.00)\end{array}$ & \begin{tabular}{|l|}
100.00 \\
$(90.00)$
\end{tabular} & $\begin{array}{l}100.00 \\
(90.00)\end{array}$ & $\begin{array}{l}100.00 \\
(90.00)\end{array}$ & $\begin{array}{l}100.00 \\
(90.00)\end{array}$ \\
\hline $\begin{array}{l}\text { Copper oxychloride } \\
(0.30 \%)\end{array}$ & $\begin{array}{l}100.00 \\
(90.00)\end{array}$ & $\begin{array}{l}100.00 \\
(90.00)\end{array}$ & \begin{tabular}{|l|}
97.22 \\
$(8338)$
\end{tabular} & $\begin{array}{l}97.22 \\
(83.38)\end{array}$ & $\begin{array}{l}97.22 \\
(83.38)\end{array}$ & $\begin{array}{l}97.22 \\
(83.38)\end{array}$ \\
\hline Check & $\begin{array}{l}0.00 \\
(0.00)\end{array}$ & $\begin{array}{l}0.00 \\
(0.00)\end{array}$ & \begin{tabular}{|l|}
0.00 \\
$(0.00)$
\end{tabular} & $\begin{array}{l}0.00 \\
(0.00)\end{array}$ & $\begin{array}{l}0.00 \\
(0.00)\end{array}$ & $\begin{array}{l}0.00 \\
(0.00)\end{array}$ \\
\hline $\mathrm{CD}$ at $5 \%$ & 1.99 & 2.10 & 2.20 & 2.59 & 2.53 & 2.92 \\
\hline
\end{tabular}

The growth inhibition of $F$. udum was found highest in $T$. viride (67.70-81.48\%) followed by $A$. niger (53.12-72.54\%) and $P$. notatum $(32.29-63.70 \%)$ at $48 \mathrm{hrs}$ to $144 \mathrm{hrs}$ incubation. Singh et al., (2010) observed maximum inhibition of radial growth of $F$. udum with $T$. viride $(83.48 \%)$ followed by $A$. niger $(52.3 \%)$ and $T$. harzianum (35.8\%). The growth inhibition of $F$. udum by culture filtrates of the test fungi is possibly attributed 
to the secretion of antibiotics by the fungi. Upadhyay and Rai (1987) on the inhibitory substances produced by the antagonists such as geodin, terricin, terric acid aspergillic acid dermadin etc.

Fungicides propiconazole $(0.15 \%)$ and copper oxychloride $(0.30 \%)$ evaluated against $F$. udum were highly effective for inhibiting the growth (100\%). Mahesh and Saifulla (2006) evaluated the contact fungicides in vitro and found copper oxychloride was the most effective, which inhibited mycelia growth $97.66 \%$ at $2000 \mathrm{ppm}$.

Out of seventeen treatments tested in vitro, propiconazole $(0.15 \%)$ was found most effective in inhibiting radial growth of the pathogen followed by copper oxychloride, Garlic, Garlic + Tulsi, Tulsi, T. viride, Ginger + Mehandi, Neem, Onion, A. niger, Ginger, Onion + Beal, Mehandi, Beal, Neem + Marigold, P. notatum and Marigold over the check.

\section{Efficacy of plant extracts, chemicals and bio-agents in vivo}

Plant extracts (10\% concentration), T. viride, A. niger, $P$. notatum (each $4 \mathrm{~g} / \mathrm{kg}$ seed). Propiconazole $(0.15 \%)$ and Copper oxychloride $(0.30 \%)$ were used for the test in vivo to find out the effectiveness against pigeonpea wilt (Pot experiment) at 45, 60, 75, 90, 105 and 120 days after sowing. On the basis of screening results in vitro. $10 \%$ concentration of plant extracts were amended in sterilized soil $(\mathrm{w} / \mathrm{v})$. The disease was controlled significantly in all plant extracts (Table 3). The effectiveness of more than $50 \%$ was recorded in all treatment at 45 and 60 days after sowing.

The highest percent disease control was 97.22 to 94.44 in Garlic and lowest 58.33 to 49.44 in Marigold. At 75 days after sowing, $50 \%$ disease control was recorded in Garlic, Garlic
+ Tulsi, Tulsi, Ginger + Mehandi, Neem, Onion and Ginger, but only Garlic $(66.66 \%)$, Garlic + Tulsi $(58.37 \%)$ and Tulsi $(50.00 \%)$ gave more than $50 \%$ disease control at 120 days after sowing.

Effectiveness of Garlic and Neem as biofungicides has already been reported by many works against different fungi. Chandra and Singh (2005) described that plant extracts of A. sativum, A. indica and Calotropis procera significantly reduced the wilt incidence in Cicer arietinum.

Among the bio-agents seed treatment with $T$. viride had controlled maximum wilt incidence 47.22 to $88.88 \%$ followed by A. niger (41.66 to $77.78 \%$ ) and $P$. notatum (25.00 to $61.11 \%$ ). It was significantly higher than the check up to 120 days after sowing. Adoms (1990) has been reviewed the fungal bio-agent like spp. of $T$. viride, Penicillium and Aspergillus against the successful control of Fusarium wilt.

Propiconazole $(0.15 \%)$ and Copper oxychloride $(0.30 \%)$ applied in the sterilized soil before the sowing of seed. Both fungicides were highly effective in reducing the disease incidence.

The effectiveness was $100 \%$ in propiconazole and 97.22 to $100 \%$ in copper oxychloride. Mehta et al., (2010) have also reported the high concentration of propiconazole (1000 $\& 1500 \mathrm{ppm})$ and copper oxychloride inhibited cent percent mycelium growth of $F$. udum. The percent inhibition was decrease in copper oxychloride with increase in period of fungicides application.

Among the seventeen treatments the seed germination in fungicides were found significantly superior than the bioagents and plant extracts. The bioagents were also superior over the plants extracts. 


\section{References}

Adoms, B., 1990. Biologocal control of Fusarium wilt disease of pigeonpea, $J$. Plant Pathology, 18(5) 279-283.

Ali, M., 2007. Global pulse production trends and challenges.National symposium on legumes' for ecological Sustainability: emerging challenges and approtunities. IIPR Kanpur: 7-10.

Ali, M., and S. Kumar. 2005. Advances in pigeonpea research. IIPR Kanpur pp-3.

Anonymous, 2011. Annual report- All India Cordinated Research Project on pigeonpea, IIPR Kanpur pp 18-32.

Chandra, H., and S. Singh. 2005. Control of chickpea wilt using bioagents and plant extracts. Indian J. Agric. Sci., 75 (2), 115116.

Devi, S., and M. Chhelry. 2012. Effect of extracts of some medicinal plants on the growth of Fusarium sp. J. Mycol. Plant Pathology, 26:110.

Dhingra, O.D., and J.B. Sinclair 1995. Basic Plant Pathology Methods, $2^{\text {nd }}$ Ed, CRC Press London. 434pp.

Joseph, B., M. Dar and V. Kumar. 2008. Bioefficacy of plant extracts to control $F$. solani of brinjal wilt, Global J. of Biotechnology, 3(2):56-59.

Kannaiyan, J., Y.L. Nene and M.V. Reddy. 1981. Survival of pigeonpea wilt Fusarium in Vertisols and Alfisols. In proceeding of the International workshop on pigeonpea, ICRISAT, Patancheru, A.P., India, 15-19 Dec.1980, 2:291-295.

Khune, R., 1990. Biological control of soil borne plant pathogens. Indian J. of Mycol.
Plant Pathology.17:1-9.

Lyon, G.D., T. Beglinski and A.C. Newton. 1995. Novel disease control compound: the potential to 'immunise' plants against infection. Plant Pathology 44:407-427.

Mahesh, M., and S. Muhammad. 2006. Evaluation of bioagents fungicides against F. udum Butler under in vitro condition. Environment and Ecology, 24(3) 824-827.

Mehta, A.N., H.L. Chauhan, K.V. Makwara, N.M. Ghel and S.L. Patel. 2010. Bio efficacy of phytoextrect, antagonist and fungicides against $F$. udum incident of pigeonpea wilt. Journal of Plant Disease Science, 5 (1):56-60.

Okigbo, R.N., 2004. A review of botanicals control methods for post-harvest yams (Dioscorea spp) in storage in South Eastern Nigeria, KMITL Sci. J. 4 (1), 207215.

Reddy, M.V., S.B. Sharma and Y.L. Nene 1990. The pigeonpea, in: pigeonpea disease management (Abstract) International, 1990, ICRISAT Patancheru, A.P., 304pp.

Singh, P.K., A. Khan, R. Gogai and R.K. Jaiswal, 2010. Plant leaf extracts and bioagents for eco-friendly management leaf wilt of pigeonpea caused by $F$. udum. Indian Pytopathology, 63(3): 343- 344.

Singh, R.S., H.S. Chaube and B. Rai. 1996. Selective agar media for the isolation of fungi. Indian Journal of Mycology and Plant Pathology, 3:67-70.

Upadhyay, R.S., and B. Rai. 1987. Studies on antagonism between $F$. udum Butler and root region microflora of pigeonpea. Plant and Soil 101: 79-93.

\section{How to cite this article:}

Sandeep Kumar, Divya Singh, Jay Kumar Yadav, Susheel Kumar and Sandeep Kumar Verma. 2017. Efficacy of Plant Extracts, Bioagents and Fungicides against Fusarium udum Causing Pigeonpea Wilt. Int.J.Curr.Microbiol.App.Sci. 6(9): 2652-2660.

doi: https://doi.org/10.20546/ijcmas.2017.609.327 